\title{
Estimação Integrada de Estados e Topologia Baseada em Informação A Priori sobre Status de Dispositivos Chaveáveis
}

\author{
Victor Freitas, Antonio Simões Costa, Fellow, IEEE \\ Departamento de Engenharia Elétrica, Grupo de Sistema de Potência \\ Universidade Federal de Santa Catarina, Florianópolis, SC, Brasil \\ victor.silva@posgrad.ufsc.br simoes@labspot.ufsc.br
}

\begin{abstract}
Resumo-Este artigo propõe um método para a estimação conjunta das variáveis de estado e da topologia da rede no contexto da modelagem em tempo real do sistema elétrico de potência. A proposta considera a representação de determinadas regiões do sistema no nível de seção de barra, explicitando os dispositivos chaveáveis nelas contidos. As informações sobre os status de disjuntores e chaves são tratadas como informação $a$ priori sobre a topologia, a ser processada por um estimador de estados ortogonal especializado, tendo como base as rotações rápidas de Givens. Além das tensões complexas nas barras, este estimador determina a topologia da rede com base nas medidas analógicas disponíveis, o que permite validar ou corrigir a topologia inicialmente presumida. $O$ método também possibilita o processamento de erros grosseiros em medidas analógicas. A metodologia proposta é avaliada e validada através de estudos de casos conduzidos com o sistema-teste de 30 barras do IEEE.

Index Terms-Modelagem em tempo real de sistemas elétricos de potência; Estimação de estados e topologia; Rotações de Givens.
\end{abstract}

\section{INTRODUÇÃO}

A operação em tempo real de Sistemas Elétricos de Potência (SEP) tem como principal objetivo garantir o suprimento da energia elétrica aos consumidores, prezando pela qualidade e economia de serviço. O Estimador de Estados apresenta-se no topo da cadeia de aplicativos da modelagem em tempo real, e se constitui na ferramenta básica para subsidiar as ações do Operador da rede elétrica. Do ponto de vista deste operador, o correto conhecimento da topologia da rede elétrica é crucial para assegurar decisões de controle confiáveis para o bom desempenho e efetividade da operação. Neste sentido, informações errôneas relacionadas à topologia geram impactos significativos capazes de comprometer os resultados da estimação de estados e por conseguinte as decisões do operador.

No início dos anos noventa, diversos estudos foram direcionados para desenvolver métodos que permitissem a estimação/validação da topologia da rede. A principal ferramenta utilizada é representação explícita do modelo físico da rede elétrica, baseada no nível de seção de barra $[1,2]$, isto é, disjuntores e chaves seccionadoras são modelados e inseridos no processo de estimação de estados [2, 3].

A topologia da rede é usualmente fornecida pelo Configurador de Redes, cuja saída são medidas digitais relacionadas aos status dos dispositivos chaveáveis. Este processo considera que, em princípio, as informações sobre os status de chaves e disjuntores estão isentas de erros. Contudo, deve-se considerar que os erros nas medidas digitais, embora menos frequentes que no caso das medidas analógicas, podem ocorrer. Desta forma, surge a necessidade de estimar não somente os estados, mas também a topologia da rede elétrica, para permitir a validação ou correção da topologia inicialmente presumida. Alguns estudos, como apresentados em [3, 4], abordam a problemática como um processo disjunto. Por outro lado, as contribuições propostas em [5-7] conduzem a estimação de estados e da topologia de forma simultânea, empregando funções bi-objetivos ou cadeias de redes neurais autoassociativas.

Este artigo baseia-se na modelagem de partes da rede elétrica no nível de seção de barra consideradas suspeitas de conter erros de modelagem. Adicionalmente, a proposta deste trabalho reconhece que informações sobre a topologia da rede estão contidas intrinsecamente nas medidas analógicas disponíveis. Desta forma, o estudo objetiva estimar os status dos disjuntores de uma determinada região suspeita de conter erros de modelagem, extraindo, das medidas analógicas, as informações sobre a topologia enquanto estima simultaneamente as tensões nodais do sistema. Para tal, a saída do Configurador de Redes é tratada como informação a priori da topologia, a qual é inserida no processo de Estimação Integrada de Estados e Topologia (EIET). A solução da EIET é obtida mediante um algoritmo baseado nas rotações ortogonais de Givens, capaz de processar informações a priori sem custo computacional adicional. De forma complementar, técnicas de identificação de zonas de anomalia $[8,9]$ são aplicadas para a diminuição da dimensão do problema.

Umas das características do método proposto é a natureza quadrática da formulação do problema, o que preserva as propriedades estatísticas das informações a priori. Isto é importante para a elaboração de testes estatísticos conclusivos capazes de detectar tanto a presença de erros em medidas analógicas quanto erros na configuração topológica da rede elétrica. Assim, de forma complementar, aborda-se também neste artigo o processamento e análise de erros grosseiros em medidas analógicas, bem como a implementação de testes de hipóteses para sua detecção e identificação.

O artigo é organizado da seguinte forma. A Seção II apresenta uma revisão dos princípios básicos da estimação de estados no nível de seção de barra, introduz a consideração de informações a priori e descreve o método de solução por um algoritmo ortogonal. A apresentação da formulação 
do problema de EIET é descrito na Seção III. As Seções IV e V descrevem, respectivamente, os procedimentos para a estimação e validação da topologia da rede e o processamento de erros grosseiros. Os resultados das simulações numéricas com o sistema-teste de 30 barras do IEEE são apresentados na Seção VI, seguida pelas conclusões finais sumarizadas na Seção VII.

\section{EstimaÇÃo de Estados no Nível de SeÇÃo de BARRA}

\section{A. Expansão do Modelo Convencional}

Neste trabalho, parte-se do pressuposto de que, a partir da estimação de estados convencional realizada com base no modelo barra-ramo, foram detectados erros de modelagem em uma certa região da rede, denominada zona de anomalia [9] (ou "bad data pocket", [2]). Esta região é então expandida de modo a evitar a ocorrência de ramos radiais, gerando o que será referido neste artigo como sub-rede relevante. A sub-rede relevante é então modelada no nível de seção de barra, no qual todos os disjuntores são explicitamente representados. Desta forma, os fluxos ativo e reativo através desses ramos chaveáveis são incluídos como novas variáveis de estado ao problema de estimação [1], e são estimados conjuntamente com as tensões nodais do sistema. Neste artigo, os estados referentes as tensões complexas são denominados estados nodais, enquanto os fluxos nos ramos chaveáveis são denominados estados de fluxo. Considerando $N$ nós no sistema e $n_{d}$ disjuntores modelados, o número de variáveis de estado a serem estimadas passa ser $n=2 N+2 n_{d}-1$.

A modelagem do status de um disjuntor entre os nós $i$ e $j$ é realizada da seguinte forma: se o ramo estiver fechado a diferença da tensão complexa entre $i$ e $j$ é igual a zero, isto é:

$$
\delta_{i}-\delta_{j}=0 \quad ; \quad V_{i}-V_{j}=0 .
$$

Por outro lado, se o ramo estiver aberto os fluxos sobre o dispositivo são nulos, logo:

$$
p_{i j}=0 \quad ; \quad q_{i j}=0 .
$$

As relações em (1) e (2) formam as condições operacionais do sistema, representadas por:

$$
h_{o}=0 .
$$

Outras configurações físicas da rede como nós de injeção nula devem também ser modeladas como restrições de igualdade, denominadas como restrições estruturais e denotadas por:

$$
h_{s}=0 \text {. }
$$

Finalmente, o modelo de medição adotado que relaciona as $m$ medidas com as $n$ variáveis de estado é dado por:

$$
\begin{aligned}
& \boldsymbol{z}_{\boldsymbol{m}}=\boldsymbol{h}_{\boldsymbol{m}}(\boldsymbol{x})+\boldsymbol{\eta}_{\boldsymbol{m}} \\
& E\left\{\boldsymbol{\eta}_{\boldsymbol{m}}\right\}=\mathbf{0} \quad ; \quad E\left\{\boldsymbol{\eta}_{\boldsymbol{m}} \boldsymbol{\eta}_{\boldsymbol{m}}^{T}\right\}=\boldsymbol{R}_{\boldsymbol{m}}
\end{aligned}
$$

onde $\boldsymbol{h}_{\boldsymbol{m}}(\boldsymbol{x})$ é o vetor $(m \times 1)$ de funções não lineares e $\boldsymbol{\eta}_{\boldsymbol{m}}$ o vetor $(m \times 1)$ de erros de medição, com média zero e elementos não correlacionados, o que define uma matriz de covariância $\boldsymbol{R}_{\boldsymbol{m}}$ diagonal e de ordem $(m \times m)$.

\section{B. Estimação de Estados em Sistemas de Potência com Infor-} mação dos Estados A Priori

O conhecimento a priori de valores para as variáveis de estado do sistema, denotados por $\bar{x}$, pode ser inserido no problema com a implementação de um termo quadrático extra no critério dos mínimos quadrados ponderados [10, 11], e restrições de igualdade podem ser consideradas como pseudomedidas de alta precisão. Desta forma, o problema de otimização passa a ser formulado como:

$$
\begin{array}{ll}
\text { Min. } & J(\hat{\boldsymbol{x}})=\boldsymbol{r}^{T} \boldsymbol{R}^{-1} \boldsymbol{r}+(\hat{\boldsymbol{x}}-\overline{\boldsymbol{x}})^{T} \boldsymbol{\Sigma}^{-1}(\hat{\boldsymbol{x}}-\overline{\boldsymbol{x}}) \\
\text { Sujeito a } & \boldsymbol{r}=\boldsymbol{z}-\boldsymbol{h}(\hat{\boldsymbol{x}})
\end{array}
$$

onde

$$
\begin{aligned}
& \boldsymbol{z}=\left[\boldsymbol{z}_{\boldsymbol{m}}^{T}, \mathbf{0}\right]^{T} ; \boldsymbol{h}(\hat{\boldsymbol{x}})=\left[\boldsymbol{h}_{\boldsymbol{m}}(\hat{\boldsymbol{x}})^{T}, \boldsymbol{h}_{\boldsymbol{s}}(\hat{\boldsymbol{x}})^{T}\right]^{T} \\
& \boldsymbol{R}=\operatorname{diag}\left\{\boldsymbol{R}_{\boldsymbol{m}}, \boldsymbol{R}_{\boldsymbol{s}}\right\} .
\end{aligned}
$$

Em (6), $\boldsymbol{\Sigma}$ é matriz de covariância dos valores dos estados $a$ priori $\overline{\boldsymbol{x}}$ e $\boldsymbol{R}_{\boldsymbol{s}}$ é a matriz diagonal de covariância das restrições estruturais, cujos elementos devem ser pelo menos uma ordem de magnitude menor que a variância típica das medidas [12]. Adicionalmente, as condições operacionais, definidas em (3), são dadas como uma topologia presumida e tratadas como informação a priori. Tal abordagem será detalhada na Seção III.

A solução do problema em (6) pode ser obtida através do método de Gauss-Newton, que recai na versão estendida da Equação Normal [13]:

$$
\left[\boldsymbol{H}^{T} \boldsymbol{R}^{-1} \boldsymbol{H}+\boldsymbol{\Sigma}^{-1}\right] \boldsymbol{\Delta} \boldsymbol{x}=\boldsymbol{H}^{T} \boldsymbol{R}^{-1} \boldsymbol{\Delta} \boldsymbol{z}+\boldsymbol{\Sigma}^{-1} \boldsymbol{\Delta} \overline{\boldsymbol{x}}
$$

onde $\boldsymbol{H}$ é a matriz Jacobiana de $\boldsymbol{h}(\boldsymbol{x})$ computada em um dado ponto $\boldsymbol{x}^{k}, \boldsymbol{\Delta} \boldsymbol{z}=\boldsymbol{z}-\boldsymbol{h}\left(\boldsymbol{x}^{k}\right)$ e $\boldsymbol{\Delta} \overline{\boldsymbol{x}}=\overline{\boldsymbol{x}}-\boldsymbol{x}^{k}$. Portanto, a estimação de estados recai em um processo iterativo em que cada iteração os estados são atualizados de acordo com (9), até que a norma infinta de $\boldsymbol{\Delta} \boldsymbol{x}$ seja menor que uma tolerância pré-especificada.

$$
\boldsymbol{x}^{(k+1)}=\boldsymbol{x}^{k}+\boldsymbol{\Delta} \boldsymbol{x}
$$

\section{Solução via Rotações Rápidas de Givens (3M)}

A utilização de métodos ortogonais para a solução de problemas de mínimos quadrados foi introduzida com o objetivo de melhorar a efetividade e robustez numérica dos algoritmos [14, 15]. O problema de otimização em (6) pode ser resolvido pela versão rápida das rotações ortogonais de Givens (3M) [16]. Considerando a forma linearizada das equações em (7), que empregam o plano de medição em (5), dada por $\boldsymbol{\Delta} \boldsymbol{z}=\boldsymbol{H} \boldsymbol{\Delta} \hat{\boldsymbol{x}}+\boldsymbol{\eta}$, sucessivas rotações ortogonais de Givens são aplicadas à matriz $\boldsymbol{H}$ e ao vetor $\boldsymbol{\Delta} \boldsymbol{z}$ (sendo ambas escalonadas por $\boldsymbol{R}^{-\frac{1}{2}}$ ) com o objetivo de se obter um conjunto que equações equivalentes, mas sob a forma de um sistema linear triangular superior. Desta forma, definindo a matriz ortogonal $\mathbf{Q}$ que armazena cumulativamente as rotações individuais, tem-se:

$$
\mathbf{Q}\left(\boldsymbol{R}^{-\frac{1}{2}}\left[\begin{array}{lll}
\boldsymbol{H} & \mid \boldsymbol{\Delta} \boldsymbol{z}
\end{array}\right]\right)=\left[\begin{array}{l|l}
\mathbf{U} & \mathbf{c} \\
0 & d
\end{array}\right]
$$

sendo $\mathbf{U}$ uma matriz triangular superior $(n \times n)$ e $\mathbf{c}$ um vetor $(n \times 1)$. Adicionalmente, a matriz $\mathbf{U}$ é decomposta conforme [14, 16]: 


$$
\mathbf{U}=\mathbf{D}^{\frac{1}{2}} \overline{\mathbf{U}}
$$

onde $\mathbf{D}$ é uma matriz diagonal e $\overline{\mathbf{U}}$ uma matriz triangular superior unitária. Como o vetor $\Delta z$ é considerado uma coluna extra de $\boldsymbol{H}$, isto resulta também no escalonamento dos elementos de $\mathbf{c}$, que passa então a ser denotado por $\overline{\mathbf{c}}$. Este artifício torna-se atrativo por tornar desnecessário o cálculo de raízes quadradas, pois na prática apenas $\mathbf{D}$ é efetivamente calculado $[14,16]$.

Ao final das transformações dadas por (11), pode-se obter o vetor de incrementos dos estados estimados $\boldsymbol{\Delta} \hat{\boldsymbol{x}}$ a partir da solução do sistema triangular de equações:

$$
\overline{\mathrm{U}} \boldsymbol{\Delta} \hat{\boldsymbol{x}}=\overline{\mathbf{c}} \text {. }
$$

A soma ponderada do quadrado dos resíduos é determinada diretamente a partir do escalar $d$, como subproduto do processo de estimação.

$\mathrm{Na}$ inicialização do método, os fatores de escala da matriz D podem ser vistos como pesos iniciais das variáveis de estado, isto é, o elemento $d_{j}$ é um fator de ponderação para a informação de estado a priori $\bar{x}_{j}$ [13]. Logo, os pesos $d_{j}^{(0)}$ podem ser definidos antes de qualquer medida ser processada, e são dados por:

$$
d_{j}^{(0)}=1 / \Sigma_{j j}
$$

onde $\Sigma_{j j}$ é a variância da informação a priori sobre a variável de estado $j$ e pertencente à matriz $\Sigma$.

Na prática, o problema de estimação de estados com informações a priori é inicializado como:

$$
\overline{\mathbf{U}}^{(0)} \boldsymbol{x}^{(0)}=\overline{\mathbf{c}}^{(0)}
$$

onde $\overline{\mathbf{U}}^{(0)}=\mathbf{I}$, em que $\mathbf{I}$ é uma matriz identidade de ordem $(n \times n)$, e o vetor $\overline{\mathbf{c}}^{(0)}=\overline{\boldsymbol{x}}$, onde $\overline{\boldsymbol{x}}$ contém as informações $a$ priori sobre o vetor de estados inicial [13].

Em resumo, o método das rotações de Givens $3 M$ pode facilmente incorporar informações a priori nos estágios de inicialização de variáveis sem custo computacional adicional. Este recurso pode ser utilizado para a implementação das informações a priori da topologia, que será discutido na próxima seção. Adicionalmente, destacam-se outras vantagens do método, como a associação de técnicas de deteç̧ão/identificação de erros grosseiros em medidas analógicas, assim como a utilização das matrizes resultantes das rotações, $\overline{\mathbf{U}}$ e $\mathbf{D}$ para cálculos de análises subsequentes à estimação de estados [14].

\section{EstimaÇ̃̃o INTEGRADA DE Estados E TOPOLOGIA}

A estimação da topologia proposta neste trabalho baseiase na extração das informações sobre a topologia contidas nas medidas analógicas disponíveis ao estimador de estados. Deste modo, os resultados fornecidos pelo Configurador de Redes (representados como condições operacionais definidas em (1) e (2)) são considerados como topologia presumida e inseridos no processo de estimação de estados como informação $a$ priori. As variâncias atribuídas a tais informações compõem a matriz $\Sigma$ da função-objetivo em (6). Os valores destas variâncias estabelecem a importância relativa associada às informações a priori de topologia com respeito às medidas no processo de estimação. Nesta perspectiva, defini-se os elementos de $\boldsymbol{\Sigma}$ em função da matriz de covariância dos erros de medição, $\boldsymbol{R}_{\boldsymbol{m}}$, da seguinte forma:

$$
\Sigma_{i i}=\bar{R}_{m} / k_{p}
$$

em que $\bar{R}_{m}$ é a média das variâncias das medidas, e $k_{p}$ é um número real positivo que funciona como parâmetro de calibração adicional, permitindo um ajuste fino das variâncias atribuídas à topologia. Vários testes realizados com diferentes sistemas levaram à conclusão de que valores na faixa $0,001<k_{p}<1$ asseguram bons resultados. É importante ressaltar que a convergência do processo de estimação de estados não é criticamente sensível aos valores de $k_{p}$. Por exemplo, mesmo a utilização de valores moderadamente maiores do que o limite superior do intervalo mencionado não comprometem a convergência do processo iterativo, embora se verifique um aumento gradual do número de iterações para a convergência à medida que valores mais elevados de $k_{p}$ são empregados. Neste trabalho, adota-se $k_{p}=0,02$.

Ao utilizar um algoritmo baseado nas rotações rápidas de Givens para a solução deste problema, as condições operacionais em (1) e (2) podem ser diretamente representadas na matriz inicial $\overline{\mathbf{U}}^{(0)}$, ao que é feito de forma simultânea à atribuição dos respectivos pesos à matriz $\mathbf{D}^{(0)}$. Deste modo, $\overline{\mathbf{U}}^{(0)}$ é definida de acordo com os status presumidos de cada disjuntor do sistema, de acordo com as regras descritas a seguir:

(a) Um disjuntor aberto $v$, cujos terminais são $k$ e $l$, é representado em $\overline{\mathbf{U}}^{0}$ conforme (14), impondo-se valores nulos aos estados de fluxo $p_{k l}$ e $q_{k l}$ em $\overline{\mathbf{c}}^{(0)}$. Simultaneamente, aos respectivos elementos diagonais de $\mathbf{D}^{(0)}$ são atribuídos pesos iguais a $d_{v v}=1 / \Sigma_{v v}$;

(b) Para um disjuntor fechado $\varphi$ conectando os nós $i$ e $j$, $j>i$, denota-se por $\zeta_{i}^{\delta}$ e $\zeta_{i}^{V}$ os índices correspondentes aos estados $\delta_{i}$ e $V_{i}$, respectivamente. Para a representação deste tipo de ramo, os valores da diagonal de $\overline{\mathbf{U}}^{(0)}$ permanecem unitários como em (14), e dois valores fora da diagonal, $\bar{u}_{\zeta_{i}^{\delta}, \zeta_{j}^{\delta}}$ e $\bar{u}_{\zeta_{i}^{V}, \zeta_{j}^{V}}$, são definidos como iguais a -1 , mantendo a natureza triangular superior unitária da matriz $\overline{\mathbf{U}}$. Adicionalmente, impõem-se valores nulos às posições $\zeta_{i}^{\delta}, \zeta_{j}^{\delta}, \zeta_{i}^{V}$ e $\zeta_{j}^{V}$ do vetor $\overline{\mathbf{c}}$. Finalmente, as linhas correspondentes aos pesos de $\overline{\mathbf{U}}^{(0)}$ são definidos como: $d_{\zeta_{i}^{\delta}, \zeta_{i}^{\delta}}=d_{\zeta_{i}^{V}, \zeta_{i}^{V}}=1 / \Sigma_{\varphi, \varphi}$, e $d_{\zeta_{j}^{\delta}, \zeta_{j}^{\delta}}=d_{\zeta_{j}^{V}, \zeta_{j}^{V}}=0$.

Considerando os disjuntores $v$ e $\varphi$ modelados nos itens (a) e $(b)$, respectivamente, a Figura 1 exemplifica as matrizes inicializadas no processo de estimação, levando em conta apenas os estados relacionados a estas informações a priori da topologia e seus respectivos pesos.

A definição da matriz $\overline{\mathbf{U}}^{(0)}$ acima torna-se clara quando relacionam-se as equações de modelagem dos disjuntores em (1) e (2) com a inicialização do algoritmo das rotações de Givens $3 M$ em (14). No caso (b), a definição de $\mathbf{D}$ é elucidada pelo fato de que a variância $\Sigma_{\varphi \varphi}$, dada por (15), condiz com a informação da queda de tensão nodal entre os terminais do disjuntor referenciada pelo nó $i$. Por outro lado, assume-se total incerteza sobre os estados nodais em $j$, o que tradicionalmente ocorre na estimação de estados convencional, baseada na ausência de informações a priori sobre os estados. 


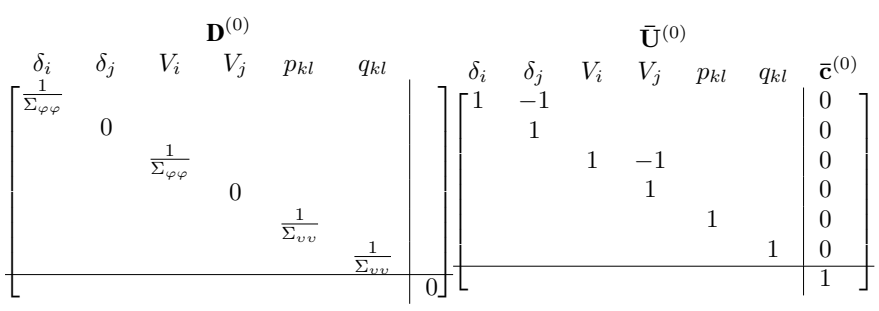

Figura 1. Matrizes de inicialização do algoritmo das rotações de Givens $3 M$ com informação a priori dos disjuntores $v$ e $\varphi$.

\section{EsTIMAÇÃO DA TOPOLOGIA}

O método de estimação integrada descrito na Seção III fornece, como saída, os valores das estimativas dos ângulos e magnitudes de tensão nas barras e os fluxos de potência ativa e reativa sobre os disjuntores. Desta forma, é necessário uma etapa de pós-processamento para validar ou corrigir a topologia inicialmente dada como presumida. Isto basicamente consiste na aplicação de testes de hipóteses associados ao algoritmo, definidos com base em um determinado nível de significância, como proposto em [3]. A partir do nível de significância definido e da variância do erro de estimação para os estados de fluxo no ramo $\ell$, determina-se um limiar $\varepsilon_{\text {fluxo }_{\ell}}$, o qual é utilizado para decidir se o ramo está de fato aberto ou fechado. Portanto, um disjuntor é considerado fechado se os valores, em módulo, dos estados de fluxo estimados forem maiores que os respectivos limiares $\varepsilon_{\text {fluxo }}$. Em caso contrário, o disjuntor é considerado como aberto. Após a verificação de todos os disjuntores, os resultados dos status estimados são comparados com a topologia presumida. Quando ocorre a identificação de disjuntores cujos status são presumidos erroneamente, o algoritmo realiza sua correção, e uma nova estimação integrada é realizada. O processo se repete até que não haja modificações nos status dos disjuntores da topologia presumida.

Nesta etapa, ressalta-se o uso das matrizes solução das rotações de Givens $3 M$, D e $\overline{\mathbf{U}}$, para computar a matriz de covariância dos erros de estimação dos estados [14], a qual é necessária no cálculo dos limiares $\varepsilon_{\text {fluxo }}$ de cada disjuntor.

\section{PRocessamento de ERros Grosseiros}

A capacidade de processar erros grosseiros é considerada uma das qualidades de qualquer estimador de estados, e isto se aplica ao Estimador Integrado de Estados e Topologia. Para tal, o método proposto faz uso dos seguintes princípios: $(i)$ os dados das informações a priori de topologia podem ser vistos como medidas virtuais, pois são tratadas na formulação em (6) como um termo de mínimos quadrados, ponderados pelo inverso da respectiva matriz de covariância, ou seja, um processo idêntico ao das medidas analógicas; (ii) como consequência, as propriedades estatísticas da solução são preservadas, e as ferramentas para o processamento de erros grosseiros previamente desenvolvidas para estimadores baseados no método dos mínimos quadrados são aplicadas em conexão com o estimador integrado. Adicionalmente, assume-se que o nível de redundância das medidas disponíveis seja suficiente para permitir o bom desempenho dos métodos convencionais de processamento de erros grosseiros.

O método é composto de dois estágios: detecção de erros grosseiros, baseado no teste- $J(\hat{x})$ [1], e identificação da medida errônea, baseado no teste- $\hat{b}$, aplicando um teste de hipóteses à uma estimativa de magnitude de erro da medida que apresenta o maior resíduo normalizado [1]. Este artigo utiliza as características vantajosas do método de Givens $3 M$, descritas na subseção II-C, para o processamento de erros grosseiros em medidas analógicas. Com base no estudo realizado em [14], este algoritmo permite a monitoração da evolução do valor de $J(\hat{\boldsymbol{x}})$ e a sua comparação com o limiar do qui-quadrado [1] após o processamento sequencial de cada medida.

A partir da detecção positiva de erros grosseiros indicada pelo estimador de estados pelo teste- $J(\hat{x})$, aplica-se o teste- $\hat{b}$ na medida analógica que possui o maior resíduo normalizado $\left(r_{i}^{N}\right)$. Se a estimativa do erro $\hat{b}$ para a referida medida exceder um limiar pré-especificado (usualmente 4, de acordo com [1]), conclui-se que a medida analógica é errônea, e desta forma, é removida do conjunto de medição. A remoção da medida ocorre com o reprocessamento da linha da matriz Jacobiana $\boldsymbol{H}$ referente à medida com erro grosseiro, com ponderação negativa $\left(-R_{m, i i}^{-1}\right)[14]$.

Por outro lado, se a primeira aplicação do teste- $\hat{b}$ for negativa para os erros em medidas analógicas, pode-se concluir que a detecção ocorrida no teste- $J(\hat{x})$ é devida a erros na topologia presumida ao estimador de estados, e portanto, nenhuma ação é tomada neste estágio, pois o processo de validação da topologia (ver Seção IV), é executado como próximo passo.

O estudo de caso descrito a seguir ilustra a aplicação dos procedimentos acima.

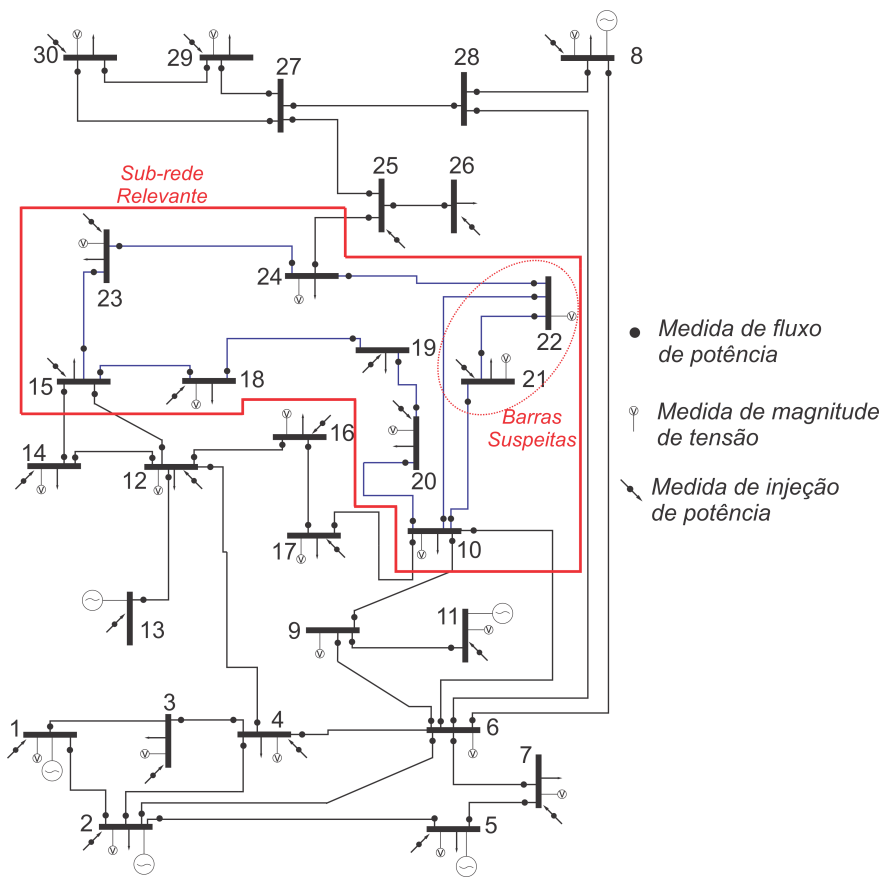

Figura 2. Sistema de 30 barras do IEEE 


\section{Simulações E Resultados Numéricos}

De modo a avaliar o desempenho da EIET, foram simulados casos utilizando o sistema-teste do IEEE de 30 barras. O sistema e o correspondente plano de medição são apresentados na Figura 2. Considerando $\mathcal{E}_{i}$ a exatidão assumida ao medidor $i$, então, as variâncias são computadas como segue:

$$
R_{m, i i}=\left(\mathcal{E}_{i} \cdot z_{m, i}\right)^{2}+\mathcal{E}_{i}^{2}
$$

conforme prática corrente, proposta em [17]. Assume-se também que a exatidão $\mathcal{E}$ das medidas de magnitude de tensão é de $3 \times 10^{-3}$ p.u., e de $2 \times 10^{-2}$ p.u. para as medidas de potência. A inclusão de erros de topologia e erros em medidas analógicas envolve as subestações 21 e 22 , consideradas como barras suspeitas (zona de anomalia). Consequentemente, determinase uma sub-rede relevante do sistema $[8,9]$, destacada também na Figura 2. As barras suspeitas são detalhadas no nível de seção de barra e apresentadas na Figura 3. Por conveniência, as subestações 21,22 e as outras barras do sub-sistema foram renumeradas, mas os números das barras originais são mostrados entre parênteses. As duas subestações são formadas por arranjos do tipo anel e disjuntor-e-meio. O modelo nãolinear da rede é implementando, permitindo a representação das quantidades de potência ativa e reativa, assim como as tensões complexas nas barras.
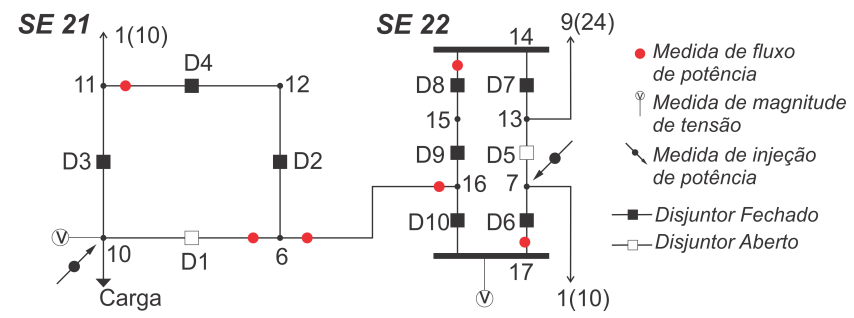

Figura 3. Sub-sistema para o sistema-teste de 30 barras

\section{A. Erro By-Pass em Subestação}

Esta subseção apresenta os resultados obtidos pelo método de estimação integrada de estados e topologia, discutido na Seção III. Neste estudo de caso, os status de três disjuntores (D5, D6 e D7, mostrados na Fig. 3) que fazem parte de um único ramo com arranjo disjuntor-e-meio, são presumidos erroneamente, ou seja, os disjuntores D6 e D7 são assumidos como abertos e o disjuntor D5 como fechado (erro by-pass). Este erro acarreta a desconexão da subestação 22 das barras 10 e 24 do sistema. Após a aplicação do algoritmo EIET, a Tabela I apresenta como os status presumidos são corrigidos e validados no processo iterativo, levando duas iterações para a convergência. A Tabela II mostra os valores dos termos que compõem a função-objetivo, computados ao final da primeira iteração (isto é, na presença de erros na topologia) e na convergência. Verifica-se que a maior contribuição do valor de $J(\hat{x})$, quando ocorre erros de topologia presumida, é proveniente do termo das informações a priori, o que fica evidente quando se compara os valores finais das duas iterações, em que o referido termo sofre redução relevante.
Tabela I

MudanÇA Dos Status Dos DisJuntores NAS ITERAÇÕES Do MÉTOdo

\begin{tabular}{|r|c|c|c|c|c|c|}
\hline Disjuntor & $D 5$ & $D 6$ & $D 7$ & $D 8$ & $D 9$ & $D 10$ \\
\hline Status Correto & 0 & 1 & 1 & 1 & 1 & 1 \\
\hline Status Presumido & 1 & 0 & 0 & 1 & 1 & 1 \\
\hline \hline Iteração 1 & $\mathbf{0}$ & $\mathbf{1}$ & $\mathbf{1}$ & 1 & 1 & 1 \\
\hline Iteração 2 & $\mathbf{0}$ & $\mathbf{1}$ & $\mathbf{1}$ & 1 & 1 & 1 \\
\hline
\end{tabular}

Tabela II

COMPONENTES DA FunÇÃO-OBJETIVO COM ERRo By-Pass DE TOPOLOGIA

\begin{tabular}{|c|c|c|}
\hline Função-Objetivo & Inicial & Final \\
\hline \hline$J(\hat{\boldsymbol{x}})$ & 5,8211 & 0,3128 \\
\hline $\boldsymbol{r}^{\boldsymbol{T}} \boldsymbol{R}^{-1} \boldsymbol{r}$ & 0,4010 & 0,3069 \\
\hline$(\hat{\boldsymbol{x}}-\overline{\boldsymbol{x}})^{\boldsymbol{T}} \boldsymbol{\Sigma}^{-1}(\hat{\boldsymbol{x}}-\overline{\boldsymbol{x}})$ & 5,4201 & 0,0059 \\
\hline
\end{tabular}

\section{B. Erros Simultâneos de Topologia e em Medidas Analógicas}

Nesta subseção são apresentados os resultados obtidos a partir do estimador integrado de estados e topologia na presença simultânea de erros na topologia presumida, fornecida pelo Configurador de Redes, e de erro grosseiro em medida analógica. Tal caso será estudado como uma situação específica da operação, na qual a medida de fluxo de potência ativa $p_{6-10}$, localizada na subestação 21 (ver Fig. 3), é simulada com erro grosseiro cuja magnitude é de 15 desvios-padrão. Da mesma forma, o disjuntor D1, cujo fluxo de potência ativa está diretamente relacionado com a medida $p_{6-10}$, é presumido erroneamente como fechado. A Tabela III apresenta o valor da medida com e sem o erro grosseiro, e a Tabela IV detalha o estágio de seu processamento. A medida portadora de erro grosseiro é detectada e identificada na segunda iteração do algoritmo das rotações de Givens $3 M$ e na primeira do laço externo da estimação integrada de estados e topologia. Como mostrado na Tabela $\mathrm{V}$, este último leva duas iterações para convergir. Após a remoção da medida com erro grosseiro, o valor da função-objetivo $J(\hat{x})$ diminui de forma notória, como pode ser visualizado também na Tabela IV. De forma complementar, já com a ausência dos efeitos do erro grosseiro no algoritmo de EIET, a etapa de correção e validação da topologia ocorre com o processo habitual, e os status dos disjuntores são estimados a partir de um conjunto de medidas sem erros grosseiros. Por fim, a Tabela VI apresenta os valores dos termos componentes da função-objetivo, computados ao final da primeira iteração (isto é, na presença da topologia incorreta, mas após a remoção da medida com erro grosseiro) e na convergência.

Tabela III

Simulação de Erro Grosseiro Em MedidA ANALógicA

\begin{tabular}{|c|c|c|}
\hline $\begin{array}{c}\text { Medida } \\
\text { Errônea }\end{array}$ & $\begin{array}{c}\text { Valor } \\
\text { Real (p.u.) }\end{array}$ & $\begin{array}{c}\text { Valor } \\
\text { Simulado (p.u.) }\end{array}$ \\
\hline \hline$p_{6-10}$ & 0,0 & 0,10619 \\
\hline
\end{tabular}

Tabela IV

Processamento de Erro Grosseiro COM AdiÇão ERro DE TOPOLOGIA

\begin{tabular}{|c|c|c|c|c|c|}
\hline $\begin{array}{c}\text { Medida } \\
\text { Identificada }\end{array}$ & $r_{i}^{N}$ & $\hat{b}$ & $\begin{array}{c}\text { Iter. } \\
\text { Givens } 3 M\end{array}$ & $\begin{array}{c}J(\hat{x}) \\
\text { Antes }\end{array}$ & $\begin{array}{c}J(\hat{x}) \\
\text { Depois }\end{array}$ \\
\hline \hline$p_{6-10}$ & 11,046 & 15,044 & 2 & 122,333 & 0,315 \\
\hline
\end{tabular}


Tabela V

Mudança Dos Status dos Disjuntores nAs ITERAÇões Do Método

\begin{tabular}{|r|c|c|c|c|}
\hline Disjuntor & $D 1$ & $D 2$ & $D 3$ & $D 4$ \\
\hline Status Correto & 0 & 1 & 1 & 1 \\
\hline Status Presumido & 1 & 1 & 1 & 1 \\
\hline \hline Iteração 1 & $\mathbf{0}$ & 1 & 1 & 1 \\
\hline Iteração 2 & $\mathbf{0}$ & 1 & 1 & 1 \\
\hline
\end{tabular}

Tabela VI

COMPONENTES DA FunÇÃO-OBJETIVO COM ERRos SimultÂnEOS NA TOPOLOGIA E MEDIDAS ANALÓgICAS

\begin{tabular}{|c|c|c|}
\hline Função-Objetivo & Inicial & Final \\
\hline \hline$J(\hat{\boldsymbol{x}})$ & 0,3142 & 0,3118 \\
\hline $\boldsymbol{r}^{\boldsymbol{T}} \boldsymbol{R}^{-1} \boldsymbol{r}$ & 0,3060 & 0,3059 \\
\hline$(\hat{\boldsymbol{x}}-\overline{\boldsymbol{x}})^{\boldsymbol{T}} \boldsymbol{\Sigma}^{-1}(\hat{\boldsymbol{x}}-\overline{\boldsymbol{x}})$ & 0,0082 & 0,0059 \\
\hline
\end{tabular}

\section{Partida Plana da Topologia}

Esta subseção apresenta os resultados do estimador proposto quando o processo de inicialização parte da completa ausência de informação sobre os status dos disjuntores da subestação, e portanto, todos são considerados como abertos (partida plana da topologia). Como mostrado na Tabela VII, o algoritmo leva duas iterações para a convergência, com o processo de estimação e correção da topologia seguido da validação na próxima iteração. Finalmente, a Tabela VIII apresenta os valores dos termos componentes da função-objetivo computadas no final da primeira (com a presença de erros de topologia) e da convergência.

Tabela VII

MudançA Dos Status dos Disjuntores nAs IteraÇões Do MÉtodo

\begin{tabular}{|}
\begin{tabular}{|r|c|c|c|c|c|c|}
\hline Disjuntor & $D 1$ & $D 2$ & $D 3$ & $D 4$ & $D 5$ & $D 6$ \\
\hline Status Correto & 0 & 1 & 1 & 1 & 0 & 1 \\
\hline Status Presumido & 0 & 0 & 0 & 0 & 0 & 0 \\
\hline \hline Iteração 1 & 0 & $\mathbf{1}$ & $\mathbf{1}$ & $\mathbf{1}$ & 0 & $\mathbf{1}$ \\
\hline Iteração 2 & 0 & $\mathbf{1}$ & $\mathbf{1}$ & $\mathbf{1}$ & 0 & $\mathbf{1}$ \\
\hline Disjuntor & $D 7$ & $D 8$ & $D 9$ & $D 10$ & \\
\hline Status Correto & 1 & 1 & 1 & 1 \\
\cline { 1 - 5 } Status Presumido & 0 & 0 & 0 & 0 \\
\hline \hline Iteração 1 & $\mathbf{1}$ & $\mathbf{1}$ & $\mathbf{1}$ & $\mathbf{1}$ \\
\hline Iteração 2 & $\mathbf{1}$ & $\mathbf{1}$ & $\mathbf{1}$ & $\mathbf{1}$ \\
\hline
\end{tabular}
\end{tabular}

Tabela VIII

Componentes da Função-ObJetivo com Partida Plana dos DISJUNTORES

\begin{tabular}{|c|c|c|}
\hline Função-Objetivo & Inicial & Final \\
\hline$J(\hat{\boldsymbol{x}})$ & 32,2720 & 0,3128 \\
\hline $\boldsymbol{r}^{\boldsymbol{T}} \boldsymbol{R}^{-1} \boldsymbol{r}$ & 1,0425 & 0,3069 \\
\hline$(\hat{\boldsymbol{x}}-\overline{\boldsymbol{x}})^{\boldsymbol{T}} \boldsymbol{\Sigma}^{-\mathbf{1}}(\hat{\boldsymbol{x}}-\overline{\boldsymbol{x}})$ & 31,2294 & 0,0059 \\
\hline
\end{tabular}

\section{CONCLUSÕES}

Este artigo propõe um algoritmo para a Estimação Integrada de Estados e Topologia com base no tratamento da topologia como informação a priori, considerando que parte do sistema é modelada no nível de seção de barra. A proposta permite a validação/correção da saída do Configurador de Redes, e assim previne a contaminação dos estados estimados por erros de topologia. Os casos apresentados neste artigo estão relacionados ao sistema do IEEE de 30 barras, na presença de níveis adequados de redundância de medidas analógicas na qual uma sub-rede relevante é determinada. Considera-se a ocorrência de anomalias de diferentes tipos, sejam eles unicamente de topologia ou com a incidência simultânea de erros grosseiros em medidas analógicas. $\mathrm{O}$ algoritmo possui boas qualidades de convergência, levando tipicamente duas iterações do laço externo para realizar o processo de correção e validação da topologia da rede. A preservação das características estatísticas viabiliza o processamento de erros grosseiros por métodos confiáveis e bem testados, baseados no critério dos mínimos quadrados. A associação com um algoritmo ortogonal permite que medidas analógicas contaminadas por erros grosseiros sejam igualmente detectadas, identificadas e removidas na segunda iteração, após a inicialização do método iterativo com base na partida plana dos estados.

\section{AGRADECIMENTOS}

Os autores agradecem o suporte financeiro do CNPq a esta pesquisa.

\section{REFERÊNCIAS}

[1] A. Monticelli, State Estimation in Electric Power Systems: A Generalized Approach, New York: Springer, 1999, p. 394.

[2] O. Alsaç, N. Vempati, B. Stott and A. Monticelli, "Generalized state estimation", IEEE Trans. Power Systems, vol. 13, pp. 1069-1075, Aug., 1998.

[3] K. A. Clements and A. Simões Costa, "Topology error identification using normalized Lagrange multipliers", IEEE Tans. Power Systems, vol. 3, pp. 347-353, May. 1998

[4] N. Vempati, C. Silva, O. Alsaç and B. Bolt, "Topoloy Estimation”, in PES IEEE, vol. 1, pp. 806-810, June, 2005.

[5] N.S. da Silva, A. Simões Costa, K.A. Clements e E. Andreoli, "Simultaneous estimation of state variables and network topology for power system real-time modeling", Electric Power Systems Research, vol. 133, pp. 338-346, Jan., 2016.

[6] E. Andreoli, A. Simões Costa and K. A. Clements, "Topology validation via simultaneous state \& topology estimation with phasor data processing capability", in 18th PSCC, pp. 18-22, Wroclaw, Aug., 2014.

[7] J. Krstulovic, V. Miranda, A. Simões Costa and J. Pereira, "Towards an auto-associative topology states estimator", IEEE Tans. Power Systems, vol. 28, No. 3. pp. 3311-3318, Aug., 2013.

[8] A. Simões Costa e J. A. Leão, "Identification of topology errors in power system state estimation", IEEE Trans. Power Systems, vol. 8, n. 4, pp. 1531-1538, Nov, 1993.

[9] A. Simões Costa, E. M. Lourenço and L. Colzani, "Reduced zone determination for topology error processing in generalized state estimation", in Power Tech IEEE, pp. 137-142, Lousanne, 2007.

[10] P. Swerling, "Modern state estimation methods from the viewpoint of the method of least squares", IEEE Trans. Automatic Control, vol. AC16, No. 6, pp. 707-719, Dec., 1971

[11] M. B. Do Coutto Filho e J. C. S. de Souza, "Forecasting-aided state estimation - Part I: panorama", IEEE Trans. Power Systems, vol. 24, n. 4, pp. 1667-1677, Nov., 2009.

[12] E. M. Lourenço, L. B. Souza and A. Simões Costa. "A unified approach for bad data and topology error identification in generalized state estimation", in 16th PSCC, pp. 18-22, Glasgow, July, 2008.

[13] A. Simões Costa, M. E. Lourenço and F. Vieira, "Topology error identification for orthogonal estimators considering a priori state information", in 15th PSCC, Liège, Belgium, session 26, paper 3, pp. 1-7, Aug. 2005

[14] A. Simões Costa and V. H. Quintana, "An orthogonal row processing algorithm for power system sequential state estimation", IEEE Transactions on Power Apparatus and Systems, vol. PAS-100, pp.3791-3800, No. 8, 1981.

[15] N. Vempati, I. W. Slutsker and W. F. Tinney, "Enhancement to Givens rotations for power system state estimation", IEEE Transactions on Power Systems, vol. 6, pp.842-849, May, 1991.

[16] W. M. Gentleman, "Least squares computations by Givens transformations without square roots", Journal of the Inst. Math. Applics., vol. 12, pp. 329-336, 1973.

[17] Dopazo J.F., Klitin O.A., Van Slick L.S.,"State calculation of power systems from line flow measurements, part II", IEEE Transactions on Power Apparatus and Systems, vol. PAS-91, pp. 145-151, Jan./Fev. 1972. 